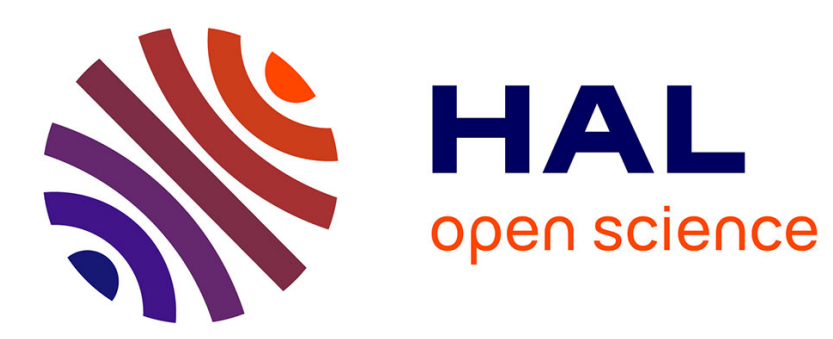

\title{
Protestantisme en migration : entre les îles Cook et la Nouvelle-Zélande
}

\author{
Gwendoline Malogne-Fer
}

\section{To cite this version:}

Gwendoline Malogne-Fer. Protestantisme en migration: entre les îles Cook et la Nouvelle-Zélande. Autrepart - Revue de sciences sociales au Sud, 2010, 56, pp.231-234. halshs-00538891

\section{HAL Id: halshs-00538891 https://shs.hal.science/halshs-00538891}

Submitted on 15 Jun 2015

HAL is a multi-disciplinary open access archive for the deposit and dissemination of scientific research documents, whether they are published or not. The documents may come from teaching and research institutions in France or abroad, or from public or private research centers.
L'archive ouverte pluridisciplinaire HAL, est destinée au dépôt et à la diffusion de documents scientifiques de niveau recherche, publiés ou non, émanant des établissements d'enseignement et de recherche français ou étrangers, des laboratoires publics ou privés. 


\title{
Protestantisme en migration : entre les îles Cook et la Nouvelle-Zélande
}

\author{
Gwendoline Malogne-Fer*
}

L'évangélisation des îles Cook $^{1}$, en Polynésie, entre 1821 (Aitutaki) et 1857 (Pukapuka), est l'œuvre conjointe des missionnaires britanniques de la London Missionary Society (LMS) et de Polynésiens convertis - dont le plus célèbre est Papehia - évangélistes et Teachers originaires des îles Sous le Vent (actuelle Polynésie française) ${ }^{2}$. Contrairement aux missionnaires britanniques qui, à partir de 1806, devaient se marier avant leur départ pour la Polynésie, les Teachers tahitiens ont pu épouser des femmes autochtones ${ }^{3}$. Le mariage de Papehia avec une fille de chef de haut rang (Ariki) de Rarotonga symbolise ainsi l'alliance originelle entre Ariki et protestantisme [Lange, 2005, p. 63-64]. En 1839, l'ouverture du Takamoa Theological College à Rarotonga permet de former les Cook Islanders qui deviendront à leur tour pasteurs ou missionnaires dans les îles de Polynésie et de Mélanésie, notamment, à partir de 1872, en Papouasie NouvelleGuinée [ibid., p. 66-77]. Proportionnellement au nombre d'habitants, ce sont les Cook Islanders qui ont fourni à la LMS le plus grand nombre de missionnaires en Océanie. Turakiare Teauariki [1996], dernier missionnaire originaire des îles Cook en Papouasie Nouvelle-Guinée, de 1963 à 1975, est devenu à son retour président de la Cook Islands Christian Church (CICC), Église congrégationaliste héritière de la LMS.

* Sociologue rattachée au Groupe Religions, Sociétés, Laïcités (Paris).

1. Ces îles, nommées ainsi depuis 1888 en l'honneur du navigateur James Cook qui les « découvrit » au cours de son deuxième voyage (1772-1775), sont composées de 15 îles polynésiennes réparties en 2 groupes : l'archipel du Nord composé d'atolls et celui du Sud composé d'îles hautes dont Rarotonga, l'île principale.

2. Ces recherches ont été réalisées dans le cadre d'un détachement au CNRS et d'une allocation post-doctorale de l'Institut Émilie du Châtelet portant sur l'accès des femmes polynésiennes au pastorat dans les églises protestantes de Nouvelle-Zélande. La méthode repose sur des entretiens semi-directifs effectués auprès de pasteurs en Nouvelle-Zélande (Auckland et Wellington) en 2005 et 2007 et à Rarotonga (îles Cook) en 2005 et 2009, la participation aux cultes et à des cérémonies particulières, la lecture des mémoires des élèves pasteurs et des archives comme le journal quotidien Cook Islands News (CIN). Les entretiens avec les pasteurs ont été privilégiés dans la mesure où j'analyse prioritairement dans cet article les transformations de l'institution religieuse et les modes de régulation du pluralisme religieux.

3. Pour une analyse historique et anthropologique de la rencontre entre missionnaires britanniques et communauté mâ'ohi à Tahiti au XIX'e siècle, voir Baré [1987]. 
La commémoration de l'arrivée de l'Évangile, le 26 octobre, jour de fête nationale, permet de célébrer l'identité nationale et chrétienne des Cook Islanders dont la spécificité est d'avoir activement participé en dehors des frontières nationales à l'évangélisation des autres îles d'Océanie. L'héritage chrétien de la nation est inscrit dans le préambule de la Constitution des îles Cook de 1965. Mais alors que la plupart des États d'Océanie accédant à l'indépendance au cours des années 1960-1970 ont érigé les «principes chrétiens » en socle fondateur de leur identité nationale, l'analyse du christianisme est restée marginale dans les études anthropologiques. En Océanie les débats autour de «l'invention de la tradition » à partir des années 1980 ont mis en évidence la centralité de la question de l' « authenticité », et avec elle celle de la permanence culturelle, en anthropologie [Friedman, 2002]. La représentation des îles du Pacifique comme espaces « hors du temps » [Thomas, 1998] et préservés des influences occidentales est restée vivace, marginalisant dans les recherches anthropologiques l'historicité des rapports sociaux, l'étude des phénomènes urbains ou du christianisme. Mais l'« authenticité » n'est pas uniquement une préoccupation des anthropologues, elle constitue pour nombre de Polynésiens une ressource argumentaire toujours disponible de (dé)légitimation des pratiques et un critère de jugement participant aux négociations identitaires notamment au sein des communautés transnationales ou en diaspora comme dans le cas des îles Cook. Analysant la production de la musique populaire des îles Cook, Alexeyeff [2004(a)] suggère, en reprenant la perspective d'Appadurai [2005], que les discussions sur le caractère (in)authentique des créations musicales illustrent les négociations identitaires au sein desquelles la production culturelle et le «travail de l'imagination » occupent une place prépondérante et contribuent à l'émergence d'une « communauté affective ». Quel est le rôle des religions chrétiennes dans la constitution de ces communautés transnationales ? Dans quelle mesure l'imbrication entre l'identité nationale et le christianisme, inscrite dans la Constitution, est-elle remise en cause ou renforcée par les migrations des Cook Islanders? Les îles Cook connaissent en effet, depuis la fin de la seconde guerre mondiale, une intensification des migrations internes (des îles éloignées vers Rarotonga, l'île principale) et internationales : au recensement de 2006 les Cook Islanders étaient 20000 aux îles Cook, 58000 en Nouvelle-Zélande et 30000 en Australie. Les migrations des Cook Islanders aux îles Cook et en Nouvelle-Zélande prennent des formes variées : migrations permanentes ou temporaires, circulations, allers-retours, elles constituent autant de déplacements englobés sous le terme de mobilité [Catarino et Morokvasic, 2005]. Le langage de la mobilité sert également à décrire les pratiques religieuses contemporaines : les figures du pèlerin et du converti inscrivent ainsi le mouvement au cœur de la modernité religieuse, la mobilité devenant un gage d'authenticité [Hervieu-Léger, 1999].

L'objectif de cet article est d'analyser les répercussions des migrations des Cook Islanders en termes de croyances et de pratiques religieuses, d'organisations ecclésiales et de redéfinition des identités culturelles dans le pays d'installation mais aussi dans le pays d'origine. 
Ces migrations s'inscrivent, en Nouvelle-Zélande comme aux îles Cook, dans un paysage religieux marqué par une forte diversification, notamment au sein du protestantisme. Cette diversification de l'offre religieuse soulève la question de l'«authenticité » des pratiques religieuses et culturelles, notamment en contexte concurrentiel et multiculturel néo-zélandais ; elle incite, aux îles Cook, les autorités religieuses et politiques à mettre en place de nouvelles modalités de gestion du pluralisme religieux.

\section{Les Cook Islanders en Nouvelle-Zélande : I'implantation des Pacific Island Churches et les ambiguiités du multiculturalisme transpolynésien}

Depuis les années 1950, les migrations des Cook Islanders vers la NouvelleZélande se sont accrues. Les Cook Islanders de la première génération étudiés par Anthony Hooper à la fin des années 1950 à Auckland constituaient une migration de travail, volontaire et a priori temporaire. Leur concentration spatiale au centre-ville d'Auckland (dans les quartiers de Ponsonby, Newton et Parnell) résultait à la fois de discriminations dans l'accès au logement et de la volonté des migrants de s'installer à proximité immédiate des personnes originaires de la même île ou du même district [Hooper, 1961]. L'intensification de ces migrations s'explique en partie par le statut constitutionnel des îles Cook. Sous protectorat britannique de 1888 à 1901 puis annexées par la NouvelleZélande en 1901, les îles Cook ont obtenu en 1964 un statut de self governance qui leur confère une autonomie politique, certaines prérogatives (monnaie et défense) restant de compétence néo-zélandaises. Ce statut se traduit par une aide annuelle de la Nouvelle-Zélande et par le maintien pour les Cook Islanders de la citoyenneté néo-zélandaise. Au recensement de 2006, les 58000 Cook Islanders de Nouvelle-Zélande continuaient de vivre quasi-exclusivement en zone urbaine, essentiellement à Auckland et Wellington (carte 1). Mais les Cook Islanders se caractérisent désormais par une proportion importante de personnes nées en Nouvelle-Zélande - $73 \%$ - et une faible maîtrise d'une langue polynésienne : seuls $16 \%$ des Cook Islanders et $5 \%$ de ceux qui sont nés en Nouvelle-Zélande parlent une langue polynésienne. Les appartenances « ethniques » multiples sont nombreuses : $47 \%$ des personnes déclarent deux ou trois appartenances, particulièrement chez les plus jeunes ${ }^{4}$. Les raisons du départ en Nouvelle-Zélande sont essentiellement liées à la poursuite d'études et la recherche d'un emploi salarié. La dimension économique des migrations ne doit cependant pas être surestimée [Morton Lee, 2004, p. 136-138] : en Nouvelle-Zélande les Cook Islanders occupent fréquemment des emplois peu qualifiés alors que les conditions de vie à Rarotonga se sont considérablement améliorées. Un autre motif, culturel et familial, permet d'expliquer l'ampleur de ces migrations.

4. Statistics New Zealand Tatauranga Aotearoa, Cook Island Maori People in New Zealand : 2006 (source : www.stats.govt.nz). 


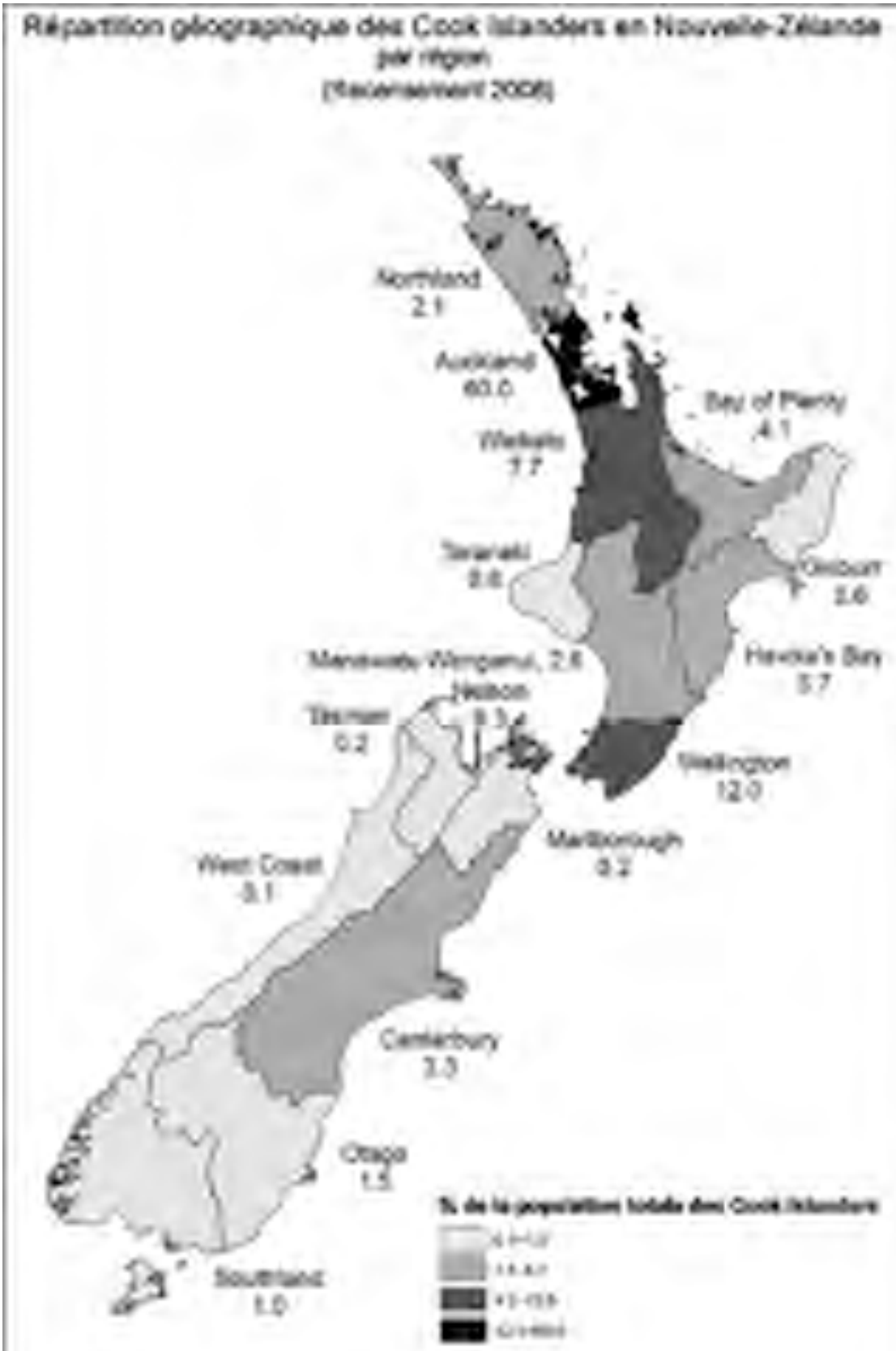

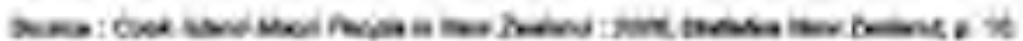


Les études anthropologiques sur les Pacific Peoples ${ }^{5}$ en Nouvelle-Zélande insistent en effet sur le maintien des liens avec les personnes restées dans les îles de Polynésie [Macpherson, Spoonley et Anae, 2001; Macpherson, Spoonley et Pearson, 2004]. Ces liens sont maintenus grâce aux échanges monétaires [Connell et Brown, 2005], d'informations (téléphone, internet, journaux télévisés, échange de cassettes) et de biens (artisanat et nourritures). Kalissa Alexeyeff a montré l'importance des échanges de nourritures entre les îles Cook et la Nouvelle-Zélande et surtout la valeur affective et symbolique de ces échanges :

«The majority of Cook Islanders live abroad (primarily in New Zealand and Australia), familial and community relationships are maintained by frequent visits to and from the Cook Islands. (...) As a result, when Cook Islanders from home visit family abroad, they take large quantities of local food with them (...) Homegrown food creates affective excess when it travels overseas. It presents the bounty of home ; the bounty of food and the bounty of sustaining loving relationships. » [Alexeyeff, 2004b, p. 68 et 70]

Les migrations s'accompagnent de représentations associées aux terres d'accueil et d'origine, qui évoluent au fil du temps : «Pour moi » - raconte le pasteur Taurangi - «quand je suis venu ici, c'est à cause de ce que j'entendais à propos de la Nouvelle-Zélande, beaucoup d'argent, une terre d'abondance, tout ce genre de choses. » ${ }^{6}$ Dans le même temps, l'attachement au pays d'origine reste particulièrement vivace chez les migrants de la première génération et se traduit par des projets de migration retour - qui ne se réalisent pas toujours, les membres de la famille proche étant également installés en Nouvelle-Zélande :

«Maintenant, je pense que je vais plutôt retourner là d'où je viens, je viens de Penrhyn, à trois heures de vol de Rarotonga, et nous pensons y retourner quand je serai pasteur à la retraite. Je ne supporte plus la vie d'aujourd'hui en NouvelleZélande. Maintenant je me rends compte que je n'ai pas une minute à moi, il faut tout le temps avoir l'œil sur la montre ! (...) Nous y sommes retournés il y a deux ans, j'ai trouvé que c'est un endroit très beau et la terre est fertile... beaucoup de choses que l'on n'a pas besoin d'acheter. Le seul problème, c'est que c'est trop loin de Rarotonga, si on est malade c'est très difficile d'aller chez le médecin, mais la vie là-bas, maintenant, je crois que c'est une île très riche ! ${ }^{7}$

Les liens entre les personnes d'une même famille dispersée géographiquement sont également maintenus grâce à l'organisation de cérémonies particulières - baptêmes, anniversaires et enterrements - le plus souvent religieuses. Ainsi en octobre 2005 la célébration - un samedi après-midi dans une salle louée à Mangere dans la banlieue d'Auckland - des 21 ans de Jenny, la fille adoptive (petite-fille) du pasteur Taurangi a rassemblé 300 Cook Islanders venus de Nouvelle-Zélande, d'Australie, des îles Cook et de Tahiti. Au cours de la cérémonie, un ancien venu

5. Le terme de Pacific Peoples désigne en Nouvelle-Zélande les migrants originaires des îles du Pacifique, le plus souvent des îles de Polynésie.

6. Entretien avec le pasteur Taurangi originaire de Penrhyn (îles Cook) de la PIC de Manukau du 21 septembre 2005.

7. Ibidem. 
de l'atoll de Penrhyn retrace la généalogie de cette famille. Les personnes rassemblées ne sont pas toutes des membres réguliers d'une église protestante de Cook Islanders, néanmoins le christianisme constitue un héritage commun qui donne une dimension religieuse non confessionnelle à cette manifestation, à travers les prières, l'interdiction d'alcool et le rappel de principes tout autant culturels que bibliques (comme le respect des anciens). Les Tivaevae, tissus cousus sous forme de patchwork, occupent une place particulière au cours de la cérémonie ${ }^{8}$. Les Tivaevae servent de dessus de lit ou de décoration dans les maisons, ils sont également fréquemment utilisés pour décorer les façades de salles omnisports ou de bâtiments loués utilisés par les Cook Islanders. Au cours de la cérémonie, les femmes s'avancent et déposent aux pieds de Jenny des Tivaevae destinés à recouvrir progressivement la jeune fille. Les Tivaevae constituent donc un support matériel permettant, en recréant un environnement polynésien, de s'approprier des lieux et un support symbolique de la réincorporation de l'individu (devenu adulte) au sein de la famille et de la communauté.

Les migrations des Pacific Peoples s’inscrivent dans un paysage religieux néozélandais marqué par des dynamiques de sécularisation - désormais les chrétiens représentent moins de la moitié de la population -, de pluralisation religieuse et d'affirmation des identités culturelles. La pratique religieuse des Cook Islanders en Nouvelle-Zélande est plus forte que celle des Pâkehâ (Néo-Zélandais d'origine européenne) mais moins importante que celles des Cook Islanders vivant aux îles Cook ${ }^{9}$. Cette fréquentation moins assidue des églises s'accompagne d'une grande mobilité religieuse. Lelei Patia, aujourd'hui pasteur de la CICC, a vécu de nombreuses années en Nouvelle-Zélande au cours desquelles il a fréquenté différentes églises chrétiennes, presbytériennes et congrégationalistes :

«J'ai grandi dans une famille qui croyait fermement en Dieu et allait à l'église. Et quand je suis venu en Nouvelle-Zélande, ça a été une période pendant laquelle je continuais à aller à l'église, mais pas aussi bien qu'avant, je commençais à adopter la manière de vivre de Nouvelle-Zélande et en tant que jeune, quelquefois je pensais à d'autres choses qu'à aller à l'église, je me suis souvent éloigné de l'église. Mais avec toujours en moi, il y avait toujours en moi une place pour Dieu. Mais quand on vient en Nouvelle-Zélande, il y a beaucoup de nouvelles choses à voir... ». ${ }^{10}$

Deux exemples permettent de mieux comprendre les reconfigurations identitaires parmi les Cook Islanders en Nouvelle-Zélande et le rôle joué par les églises et les leaders religieux : l'implantation d'Églises transpolynésiennes et la politique

8. Les épouses de missionnaires de la LMS ont introduit, au XIX ${ }^{\mathrm{e}}$ siècle, les techniques de couture et les motifs à l'origine des Tivaevae.

9. Lors du recensement de $2006,70 \%$ des Cook Islanders déclarent une affiliation religieuse et parmi eux $42 \%$ se déclarent presbytériens ou congrégationalistes. À titre de comparaison, lors du recensement de 2006 les appartenances religieuses aux îles Cook sont, par ordre d'importance : la Cook Islands Christian Church (52,6\%), l'Église catholique établie en 1894 (17\%), les Adventistes (7,5\%), les Mormons (3,7 \%), les Témoins de Jéhovah (2,1\%), les Assemblées de Dieu (3,6\%), l'Église apostolique (2\%), autres (4\%), sans religion $(3,8 \%)$, pas de réponse $(2,5 \%)$.

10. Entretien avec le pasteur Lelei Patia, pasteur de la CICC de Titikaveka, du 11 novembre 2005. 
de reconnaissance de la diversité religieuse et culturelle menée par le gouvernement néo-zélandais.

L'arrivée des Polynésiens en Nouvelle-Zélande après la seconde guerre mondiale a incité les dirigeants de la LMS à créer des paroisses polynésiennes appelées Pacific Island Churches (PIC) regroupant au sein d'une même paroisse des Polynésiens d'origine différente (Samoans, Niueans et Cook Islanders) mais partageant une culture chrétienne commune issue de la LMS ${ }^{11}$. La première PIC est implantée à Newton au centre d'Auckland à l'initiative du missionnaire Robert Challis. Au cours des années 1960-1970, suite à la création de groupes de maisons et grâce au soutien des paroissiens de Newton, plusieurs PIC sont créées dans la banlieue d'Auckland à une époque où la pression foncière au centre-ville oblige les Polynésiens à migrer en banlieue. Les PIC initialement rattachées à l'Église congrégationaliste de Nouvelle-Zélande sont intégrées en 1969 à l'Église presbytérienne de Nouvelle-Zélande, suite à la fusion entre ces deux Églises.

L'originalité de cette organisation paroissiale réside d'une part, dans la coexistence de différentes communautés polynésiennes ayant chacune un culte dans leur langue présidé par un pasteur de la même communauté linguistique, et d'autre part, dans la mise en place, à partir des années 1970, d'un culte commun en anglais destiné aux plus jeunes [Malogne-Fer, 2009a] ${ }^{12}$. Mais cette organisation paroissiale est critiquée dans la mesure où elle suppose une homogénéité culturelle problématique, les Samoans, Niueans et Cook Islanders n'ayant pas les mêmes pratiques de gestion financière ni la même compréhension du ministère pastoral. L'identité «Pacific Islanders » est ainsi régulièrement remise en cause comme le souligne Arii, étudiante au Knox College et future pasteure :

« Pour moi personnellement, l'identité Pacific Islander, c'est considéré comme un seul groupe, avec différentes îles au sein de ce même groupe. Mais je viens d'un groupe particulier et je veux que l'on me voit, qu'on dise : "C'est une femme des îles Cook". (...) La communauté Pacific Islander, ça veut dire quoi ? On dit "vous êtes Pacific Islanders" mais sans avoir la moindre idée de qui je suis, ils voient des "Brown People": ah, c'est le groupe des Pacific Islanders, mais c'est le groupe des îles Cook, pas le groupe des Pacific Islanders. C'est important, pour moi. » ${ }^{13}$

La politique néo-zélandaise se caractérise par un biculturalisme officiel - faisant de l'alliance entre Pâkehâ et Maori ${ }^{14}$ le socle de l'identité nationale néozélandaise - et un multiculturalisme de fait. Dans un contexte où les églises sont

11. Pour une analyse historique et anthropologique de la création des PIC, voir Macpherson et La'avasa [2001] et Taule'ale'ausumai [1997].

12. Sandra Fancello, analysant les pentecôtismes «indigènes » en Afrique et en Europe, notamment la Church of Pentecost du Ghana, a montré la centralité des questions linguistiques au sein de ces églises. L'usage d'une langue vernaculaire africaine comme langue de culte est une limite à l'expansion missionnaire et à la pluralisation interne de ces églises ; il permet en revanche, pour ceux qui pratiquent quotidiennement cette langue d'y voir le symbole d'une «communauté retrouvée » et pour ceux qui ne la pratiquent pas de considérer cette langue liturgique comme une «langue sacrée » [Fancello, 2008, p. 74-77].

13. Entretien avec Arii Taimataora, étudiante au Knox College à Dunedin, du 19 octobre 2005.

14. Le terme de Maori signifie « autochtone », il est réservé en Nouvelle-Zélande au peuple autochtone de Nouvelle-Zélande. Comme dans la plupart des îles de Polynésie, il est également utilisé par les Cook Islanders en particulier pour désigner leur langue (Cook Islands Maori). 
reconnues comme un acteur important de la «société civile », les leaders polynésiens religieux ont su, en créant des associations œcuméniques par île ou archipel d'origine, s'ériger en représentants légitimes des communautés polynésiennes auprès des autorités municipales et gouvernementales. Mais comme le regrettait Winnie Laban alors ministre des Pacific Affairs, les leaders religieux polynésiens ont privilégié la défense de «valeurs familiales » lors du vote des lois relatives à la légalisation de la prostitution (en 2003) et à l'établissement d'unions civiles pour les couples de même sexe (en 2004) au détriment des questions sociales et économiques tout aussi importantes ${ }^{15}$.

Pour les pasteurs rencontrés, la figure idéalisée du missionnaire est omniprésente. Le missionnaire se définit en priorité comme une personne polyglotte ou amenée à le devenir. Le pasteur Robati, de la PIC de Mangere, développe une approche nostalgique de la PIC de Newton, lieu inaugural du protestantisme des Pacific Peoples en Nouvelle-Zélande et lieu de souvenir des solidarités polynésiennes. Dans cette configuration, les pasteurs ne doivent pas restreindre leur ministère à leur communauté d'appartenance mais apprendre plusieurs langues pour redonner ses lettres de noblesse aux PIC :

«Je suis venu ici en 1966, j'avais 6 ans, je venais des îles Cook et j'ai rejoint "l'église-mère", la Newton PIC Church. J'aimais cette Église, surtout la Boys Brigade, l'école du dimanche, c'était vraiment la fraternité, je parle des Niueans, les Samoans, les Cook Islanders, il y avait une vraie unité, un véritable sens de l'unité à cette époque. Les gens avaient fait tout ce chemin depuis les îles, mais où aller ? L'Église devient comme un village, avec un sentiment de communauté, le sentiment d'être ensemble, le sentiment de partager ensemble, en ce temps-là c'était vraiment bien. (...) Mangere est vraiment un lieu différent des autres PIC, c'est assez unique. Les autres PIC ont une autre façon d'organiser le travail pastoral : le pasteur des îles Cook va travailler seulement auprès des Cook Islanders, etc. Mais ici, c'est assez unique. Le révérend Challis suivait ce modèle, il prêchait en Niuean, en Samoan, il prêchait dans la langue des îles Cook. Moi, je suis vraiment ce modèle que le révérend Challis nous a laissé. » ${ }^{16}$

L'évocation du missionnaire permet également aux rares femmes pasteures de relativiser les normes de genre en vigueur dans l'église protestante « historique » des îles Cook - la CICC n'autorise pas l'accès des femmes au pastorat [MalogneFer, 2009b]. Enfin, la figure missionnaire autorise un regard critique sur le fonctionnement traditionnel de la paroisse :

«Moi » - raconte le pasteur Tokerau Joseph - «je pense que l'un des problèmes que nous avons à Otara avec le groupe des îles Cook (...), c'est que ça n'est qu'entre nous, c'est comme si nos bras étaient fermés, nous ne voulons que nous et je ne pense pas que nous soyons très efficaces pour accueillir de nouvelles personnes, parce qu'ils ne veulent que des gens comme eux. "Cette semaine, je t’annonce la Parole, la semaine prochaine tu m'annonces la Parole, la semaine

15. Entretien du 10 novembre 2007. Pour une analyse des débats relatifs à l'ordination des gays et lesbiennes dans les églises méthodistes et presbytériennes de Nouvelle-Zélande, voir Malogne-Fer [2010].

16. Entretien avec Vaiora Robati, pasteur de la PIC de Mangere à Auckland, du 23 septembre 2005. 
d'après je t'annonce la Parole", c'est comme ça... C'est une des raisons pour lesquelles je voulais être missionnaire, parce que nous n'avons aucune nouvelle personne, personne avec qui partager la bonne nouvelle de Jésus. ${ }^{17}$

Plusieurs initiatives ont été prises par ce pasteur pour inciter les Cook Islanders à fréquenter l'église. L'organisation de «cultes de famille », en encourageant les proches des paroissiens à aller à l'église pour une cérémonie particulière, permet par exemple de concilier l'objectif missionnaire et le caractère communautaire de ces églises culturelles en transformant les familles de Cook Islanders en « champ de mission ».

Cette fragilité du modèle multiculturel trans-polynésien se traduit dans un nombre croissant de PIC par des scissions ou des fusions, aboutissant à des paroisses qui ne regroupent plus que des personnes originaires d'une même île (Niue) ou d'un même archipel (Samoa, les îles Cook). C'est donc la même dynamique - celle d'une valorisation des différences culturelles et linguistiques - qui avait permis la création des PIC qui divise aujourd'hui ceux qui s'étaient auparavant rassemblés, à la différence près que désormais l'échelle de référence s'est rétrécie en se recentrant sur l'île ou l'archipel d'origine. Analysant la gestion du multiculturalisme au sein de l'église presbytérienne, Tokerau Joseph note : «My suspicion is that they [the Presbyterian ministers] are more comfortable working among their own people, although New Zealand is becoming more and more diverse ${ }^{18}$.

Surtout, le modèle des PIC est désormais concurrencé par des Églises congrégationalistes dépendant directement des églises mères de Polynésie : c'est le cas de la Congregational Christian Church in Samoa et de la Cook Islands Christian Church (CICC) respectivement implantées en Nouvelle-Zélande depuis 1962 et 1978.

\section{La Cook Islands Christian Church : une église « historique » en transition}

Depuis les années 1970 les fortes migrations vers la Nouvelle-Zélande puis en Australie entraînent un vieillissement et une baisse de la population des îles Cook, à l'exception notable de Rarotonga, l'île principale et centre administratif, politique, culturel et économique des îles Cook, qui concentrait, en 2006, 72 \% de la population ${ }^{19}$. L'accroissement démographique à Rarotonga est dû d'une part à l'exode des archipels éloignés vers l'île principale des îles Cook et d'autre part à des migrations retour de Nouvelle-Zélande et d'Australie ${ }^{20}$. Rarotonga devient

17. Entretien avec Tokerau Joseph, pasteur de l'Église presbytérienne à Dunedin (Nouvelle-Zélande), du 14 octobre 2005.

18. Spanz March 2007.

19. Cook Islands Statistical Bulletin, Census of population and dwellings 2006 (www.stats.gov.ck).

20. Jean-Louis Rallu [1997, p. 163-190] qui a étudié les migrations retour aux îles Cook au cours des années 1990 souligne la difficulté de l'analyse lorsque les trajectoires se complexifient et que la distinction entre migrations définitives et temporaires s'amenuise. 
donc un lieu de vie, de passage et de brassage, un lieu stratégique de redéfinition de l'authenticité culturelle et religieuse. Dans ce contexte, la Cook Islands Christian Church (CICC) connaît une triple dynamique de transnationalisation, de réaffirmation culturelle et de pluralisation interne.

La Cook Islands Christian Church comptait officiellement, en 2007, 23 paroisses aux îles Cook, 20 en Nouvelle-Zélande et 13 en Australie ${ }^{21}$. L'implantation de la CICC en Nouvelle-Zélande en 1978 résulte de la prise de conscience d'une difficile intégration des Cook Islanders au sein de l'Église presbytérienne liée à des exigences académiques auxquelles les candidats au pastorat n’ont pu répondre. Le modèle des PIC apparaît en effet inadapté lorsque, par manque de pasteurs originaires des îles Cook, les Cook Islanders sont sous la responsabilité de pasteurs samoans. La réouverture du Takamoa College, le collège théologique de la CICC à Rarotonga, à la fin des années 1970, permet à l'Église de former des pasteurs chargés de créer des paroisses en NouvelleZélande, en mobilisant les réseaux de parenté. Ces paroisses, le plus souvent sans bâtiment religieux, se caractérisent par leur petite taille et depuis quelques années par une revendication identitaire qui se focalise sur une origine insulaire particulière, comme le note Vaka Ngaro, directeur du Takamoa College :

«Voilà ce qui se passe, aujourd'hui : si quelqu'un est d'Aitutaki, la communauté d'Aitutaki, ils vont préférer un pasteur de Aitutaki ; c'est le problème maintenant, nous ne voulons pas que cela se passe comme ça, mais nous ne pouvons rien faire parce que c'est pour ça que les gens préfèrent... nous n'avons pas de grandes églises, en Nouvelle-Zélande, ce sont surtout des communautés insulaires : ils ont leur propre petite communauté, avec leur propre église, et si je n'aime pas ce qu'Untel fait, je sors de cette église et j'en démarre une autre à côté... c'est pour ça que nous avons toutes ces petites églises. ${ }^{22}$

Face à l'éparpillement géographique de ses membres, la direction de la CICC a créé depuis 2005 une lettre d'information, paraissant en moyenne 8 fois par an, envoyée par e-mail (à 300 destinataires) et par courrier (500 exemplaires). Cette lettre en Cook Islands Maori, avec quelques passages en anglais, a pour objectif d'informer les paroissiens de l'actualité de l'Église (préparatifs et comptes rendus des assemblées générales et autres manifestations), des congrégations locales (rénovations et constructions de bâtiments religieux) et de ses membres. Au fil des numéros la lettre s'étoffe : de nouvelles rubriques apparaissent parmi lesquelles «l'histoire en images » et «partagez vos photos ». La lettre se termine soit par un extrait de livres retraçant l'histoire de l'évangélisation protestante aux îles Cook au XIX ${ }^{\text {e }}$ siècle soit par le portrait d'une personne, le plus souvent âgée ou décédée, dont la contribution à l'église est mise en avant. Cette lettre d'information est abondamment illustrée de photos de personnes posant devant leur église, au cours d'une cérémonie ou autour d'un repas communautaire. Elle

21. Trois paroisses sont également en cours de constitution à Napier (Nouvelle-Zélande), Brisbane et Sydney (Australie).

22. Entretien avec Vaka Ngaro, pasteur de la CICC et directeur du Takamoa College à Rarotonga, du 11 septembre 2009. 
ressemble à biens des égards à un album de famille : les dirigeants de l'Église qui visitent régulièrement les différentes églises de Nouvelle Zélande et d'Australie apparaissent sur de nombreuses photos de groupes, faisant ainsi physiquement et visuellement le lien entre les différentes «branches» de l'Église. La lettre comprend également des informations pratiques comme le dossier de candidature à l'école pastorale ou des appels de fonds. Le procédé n'est pas interactif (il est néanmoins possible d'envoyer des photos au secrétaire général de l'Église qui pourra les inclure dans la prochaine lettre) mais permet d'échanger régulièrement des informations entre les différentes CICC des îles Cook, de NouvelleZélande et d'Australie. Alan Howard a montré comment Internet pouvait jouer un rôle significatif dans le renforcement des liens déjà existants entre personnes originaires de Rotuma dispersées à travers le Pacifique [Howard, 1999]. La circulation des informations par Internet permet d'associer le renforcement des relations sociales à la déclinaison de contextes particuliers; le message envoyé le 30 octobre 2009 par Nga Mataio le secrétaire général précise ainsi comment lire cette lettre :

« Salutations dans le glorieux nom de notre Seigneur et Sauveur Jésus Christ.

J'ai le plaisir de vous adresser un exemplaire de la lettre d'informations de la CICC $\mathrm{n}^{\circ} 27$, pour un moment tranquille de lecture et peut-être pour vous distraire un peu des soucis de ce monde. Il est préférable de lire la lettre d'informations avec une noix de coco et du taro si vous êtes dans le Sud des îles Cook, avec du germe de coco (uto) et du poisson séché si vous êtes dans le Nord des îles Cook, peut-être avec un fish and chips, un sandwich au poulet et un coca si vous êtes en NouvelleZélande ou en Australie.»

Tout en multipliant les implantations paroissiales à l'étranger et en développant une communication qui déborde du cadre national, la direction de la CICC entend consolider la place du Takamoa College, à la fois centre historique de la LMS, centre politico-administratif de la CICC et surtout lieu de formation des futurs pasteurs de la CICC.

Quel que soit le lieu d'implantation, les paroisses de la CICC sont dirigées par des pasteurs formés pendant 4 ans au Takamoa College de Rarotonga. La centralisation de la formation théologique apparaît comme un axe fondamental de la politique de l'Église : les étudiants peuvent commencer ou compléter leur formation à l'étranger mais le séjour (parfois écourté) au Takamoa College demeure obligatoire. En 2006-2009, sur 12 étudiants, 10 étaient envoyés par des églises locales d'Australie (6) de Nouvelle-Zélande (4) illustrant, à travers le lien entre vocation pastorale et revendication culturelle, comment l'église peut devenir un lieu de réaffirmation identitaire dans un contexte de migrations et de multiculturalisme.

Parmi les étudiants de la dernière promotion, la maîtrise de l'anglais n'est pas satisfaisante (malgré les séjours à l'étranger) et la moyenne d'âge est élevée (43 ans). Interrogé sur ces « vocations tardives », le directeur du Takomoa College répond : 


\begin{abstract}
«Mon avis personnel, c'est que ce n'est pas un appel, parce que la plupart d'entre eux sont sans travail, ils veulent être servis plutôt que servir (rires), c'est la vérité et nous le savons, ils savent comment sont ces gens, qui viennent ici juste pour y être, juste pour être ordonné et devenir un pasteur et être comme un roi, les gens à leur service, c'est la vérité et c'est pourquoi l'Église ne grandit pas.

Il n'y a pas une période d'évaluation, vous ne pouvez pas refuser ces personnes? En fait, je crois que l'Église est trop gentille, même s'ils échouent "oh, ça ne fait rien, ils sont nos enfants, donnons leur une chance !" »
\end{abstract}

Ces vocations tardives pourraient donc être un moyen de réaliser une migration retour pour ceux qui n'ont pas réussi à s'intégrer socialement et professionnellement à l'étranger. À partir de l'étude des Tongiens immigrés en Australie Helen Morton Lee [2004, p. 144] conclut également que les migrations retour concernent prioritairement ceux qui n'arrivent plus à surmonter les difficultés financières en Australie. La candidature au collège théologique constitue en outre, grâce aux mutations pastorales qui interviennent tous les 4 ans au sein de l'Église, une promesse de mobilité.

Les entretiens effectués auprès des pasteurs de la CICC en exercice à Tahiti, à Rarotonga et en Nouvelle-Zélande, montrent que le choix du ministère pastoral s'inscrit dans une trajectoire personnelle déjà marquée par la migration. La vocation pastorale offre une possibilité de poursuivre une mobilité géographique et d'envisager une reconversion professionnelle. La figure du missionnaire sert de trame narrative aux récits biographiques : elle permet de donner un sens aux migrations antérieures à la vocation pastorale (poursuite de scolarité à Rarotonga ou recherche d'un emploi en Nouvelle-Zélande) en transformant ces dernières en étapes préparatoires au ministère pastoral, la mobilité devenant une compétence qui pourra être ultérieurement mise à profit ${ }^{23}$.

La CICC promeut le Cook Islands Maori comme langue de culte et d'activités paroissiales. La présence de nombreux touristes aux îles Cook aurait pu mettre à mal cette politique linguistique et culturelle de la CICC. Pourtant les dirigeants de l'Église voient dans le tourisme un raison supplémentaire de conserver leur «authenticité », notamment l'usage du Cook Islands Maori comme langue de culte :

«La plupart des touristes viennent pour voir et vivre le culte tel que nous le faisons ici et beaucoup d'entre eux veulent entendre nos hymnes traditionnels et nos vieux chants. Nous n'avons pas vraiment besoin de changer, la plupart des touristes qui viennent, ils l'acceptent simplement comme il est, parce qu'ils veulent voir comment nous louons Dieu à notre façon $» .{ }^{24}$

23. Véronique Boyer, à partir de l'étude des protestantismes évangéliques en Amazonie brésilienne et de la figure de l'évangéliste, montre de façon sensiblement comparable comment l'engagement religieux donne un sens aux migrations antérieures marquées par l'extrême pauvreté et la précarité : «La nouvelle appartenance religieuse incite ainsi à la réinterprétation d'une trajectoire de migration, banale et mondaine, en un parcours tout aussi accidenté mais néanmoins défini comme exceptionnel et religieux. Elle autorise une relecture acceptable des événements passés autant qu'elle constitue une justification à l'action ». [Boyer, 2008, p. 141].

24. Entretien avec Lelei Patia, pasteur de la CICC de Titikaveka du 11 novembre 2005. 
L'essor touristique n'est pas perçu comme une menace pour l'identité culturelle des Cook Islanders. Jeffrey Sissons explique ce paradoxe en mettant en évidence les liens entre l'essor du nationalisme des années 1960, dans le sillage de l'accession à l'indépendance, et le développement touristique des îles Cook destiné à consolider, au niveau économique, cette indépendance politique :

«Building a nation meant building a tourist destination, and building a tourist destination meant cultivating a culture (...) Participation in nationhood increasingly means participation in the tourist industry. The rhetoric of nationhood, which formerly emphasized the values of togetherness and progress, increasingly celebrates a more marketable ethnic pride and cultural heritage. »

[Sissons, 1997, p. 177 et 186]

La présence des touristes n'entraîne pas de profondes modifications dans le déroulement des cultes, les pasteurs admettent néanmoins faire un bref résumé de leur prédication en anglais pour être compris à la fois par les étrangers et les « jeunes » de Rarotonga qui ont une maîtrise insuffisante du Cook Islands Maori. Subrepticement la question linguistique laisse entrevoir, au-delà des clivages générationnels, une diversité des attentes paroissiales qui nécessite une gestion institutionnelle de cette pluralisation interne.

Outre la création de nombreuses paroisses à l'étranger, la CICC se singularise à Rarotonga par un fort courant évangélique qui se caractérise, pour ses membres, par l'expérience de la conversion (born again), vécue comme l'établissement d'une relation personnelle avec Jésus-Christ, le baptême d'adulte par immersion formalisant cet engagement. L'influence de ce courant évangélique au sein de la CICC s'analyse à trois niveaux : institutionnel, individuel et ministériel. Depuis 20 ans la question du baptême par immersion (au lieu du baptême par aspersion) est largement débattue au sein de l'institution ecclésiale. La direction de l'Église interdit aux pasteurs d'organiser ostensiblement cette cérémonie tout en proposant aux membres d'église qui le souhaitent de faire appel aux pasteurs des Assemblées de Dieu (pentecôtistes), l'essentiel étant qu'ils retournent ensuite dans leur église d'origine. Alors qu'en Nouvelle-Zélande le type de baptême (immersion/aspersion) construit une frontière symbolique autour de laquelle doivent se positionner les différentes Églises protestantes, aux îles Cook, le positionnement de la CICC en tant qu' «Église historique » lui permet de relativiser les clivages théologiques intraprotestants et de proposer une gestion originale de cette pluralisation interne.

Au niveau individuel, la conversion permet de donner un autre sens à sa vie, sans changer de lieu. Analysant l'essor des moyens de communication et d'information depuis quarante ans à Rarotonga - les émissions radiophoniques à partir des années 1960, la circulation des cassettes vidéo à partir des années 1970 et l'apparition de la télévision et des programmes néo-zélandais et nord-américain en 1989 - Ron et Marjorie Crocombe en concluent :

«Migration is not only physical - it can be intellectual too. Some Cook Islanders abroad retain strong ties of thought and action to the homeland, while some young 
people who have never left Cook Islands try to live abroad in their minds, using models seen on film and video in their own imagination. »

[2002, p. 13]

De façon sensiblement comparable, la conversion peut être vécue comme un voyage intérieur. Le «converti de l'intérieur [est] celui qui découvre ou redécouvre une identité religieuse demeurée jusque-là formelle, ou vécue à minima de façon purement conformiste »[Hervieu-Léger, 1999, p. 124]. Les récits de conversion relatent la mise en ordre d'une vie dissolue par l'alcool, l'accomplissement personnel mais aussi la fidélité renouvelée à l'église d'appartenance des parents. Le converti de l'intérieur, en combinant religion choisie et héritée, revendique ainsi une religion doublement « authentique ».

Enfin au niveau ministériel, le courant évangélique exprime la permanence dans l'imaginaire collectif de la figure idéal-typique du missionnaire. Mais le missionnaire contemporain ne part plus convertir les païens en Papouasie Nouvelle-Guinée, il reconvertit les Cook Islanders de l'intérieur. Cette reconfiguration du champ missionnaire - également liée à l'activisme d'organisations missionnaires internationales comme Jeunesse en Mission, présente en Nouvelle-Zélande depuis 1967 et à Rarotonga depuis 1989 - s'accompagne d'une redéfinition des identités culturelles au service de la mission :

« La culture mise en scène, tout en demeurant l'expression d'une identité singulière, n'est plus pour autant le signe d'une altérité indépassable : elle est désormais un moyen de communication, la manière à la fois la plus efficace et la plus "authentique" de se présenter aux autres. Autrement dit, la diversité culturelle devient une sorte de lieu commun permettant d'engager le dialogue et l'action missionnaire. » [Fer, 2010, p. 116]

Ces deux dynamiques missionnaires, historiques et contemporaines, en Nouvelle-Zélande et à Rarotonga, doivent se renforcer l'une l'autre. C'est en effet en son centre que le protestantisme historique est menacé par la concurrence de nouvelles églises indépendantes.

\section{L'essor de nouvelles églises protestantes à Rarotonga : les limites du pluralisme religieux}

À cette forte diversité interne à la CICC s'ajoute depuis une vingtaine d'années une multiplication d'églises indépendantes, de sensibilités pentecôtiste, charismatique ou évangélique. La recension spatiale des églises met en évidence une forte diversification de l'offre religieuse : l'île de Rarotonga d'une circonférence de 31 kilomètres compte, établis le long de la route de ceinture, 31 lieux de culte (carte 2). Outre la création des assemblées de Dieu au cours des années $1970^{25}$, le paysage protestant s'est, à Rarotonga, profondément diversifié ${ }^{26}$. Ces nouvelles

25. Les Assemblées de Dieu ont quatre églises à Rarotonga et quatre églises dans les îles à Aitutaki, Mitiaro, Mangaia et Manihiki (CIN, 21/09/07).

26. Ce processus de fragmentation et de scission structurelle en protestantisme évangélique se nourrit de la concurrence, avivée en période d'implantation d'une nouvelle église, aux postes à responsabilité et 
églises résultent à la fois de l'activité des réseaux évangéliques internationaux et de scissions au sein de la CICC et des Assemblées de Dieu; elles mettent à mal les modes de régulation du pluralisme religieux négociés au sein du Religious Advisory Council (RAC), comité religieux chargé de conseiller les autorités politiques.

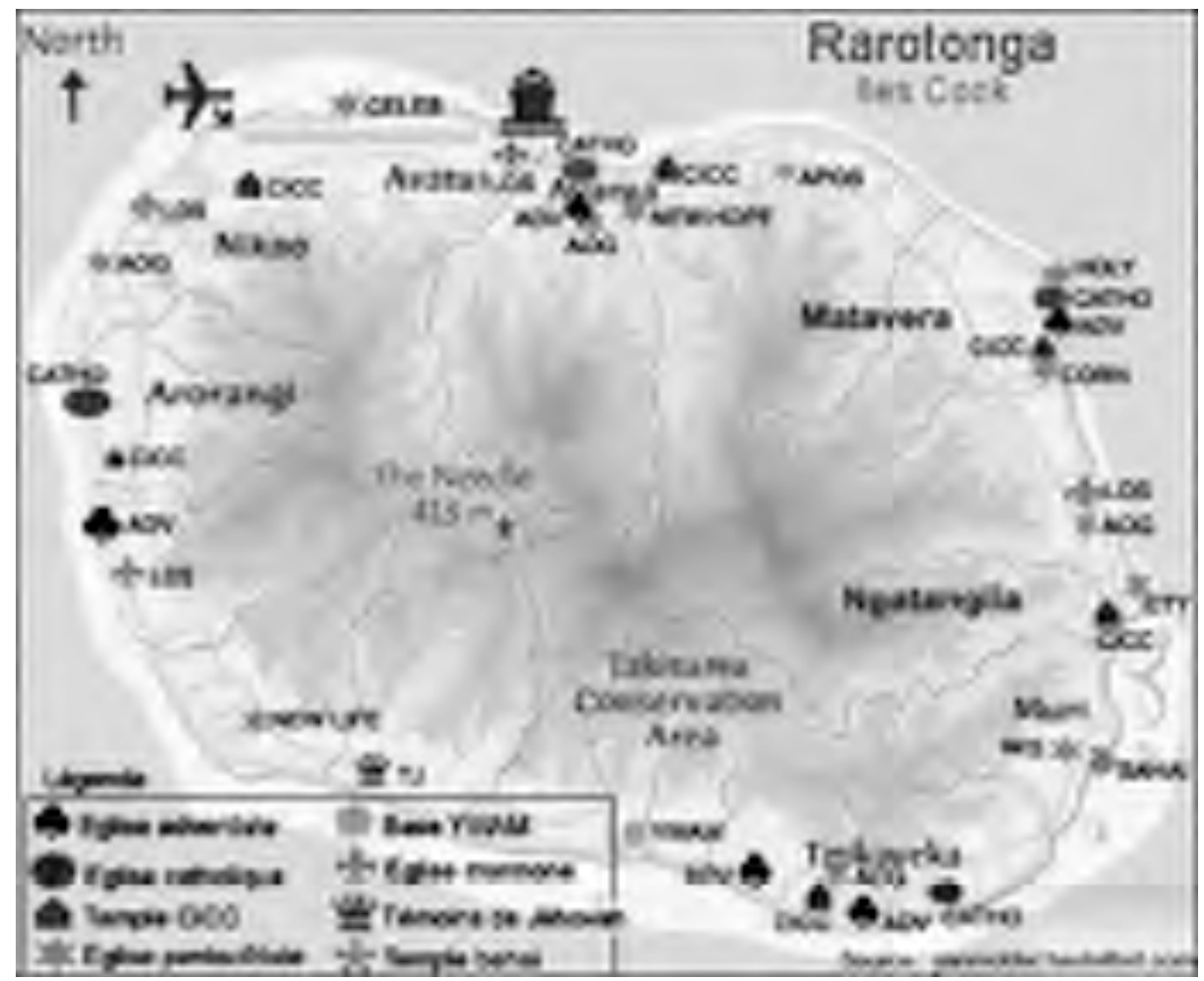

Il n'est pas possible ici de retracer dans le détail les conditions d'émergence de toutes ces nouvelles églises protestantes ${ }^{27}$. Deux exemples, l'Église apostolique créée par un pasteur de la CICC en 1988 et Celebration on the Rock, dernière église implantée sur l'île en 2009, permettront de mieux comprendre l'imbrication de logiques locales et de dynamiques transnationales à l'origine de la création de ces églises : dans le premier cas une église locale est intégrée à un réseau mondial, dans le second cas les dirigeants d'un réseau international d'églises décident de

des allers-retours des leaders religieux qui cherchent à asseoir leur autorité religieuse au-delà d'une seule congrégation locale. Or ces allers-retours fournissent la trame de nombreux conflits : c'est lors d'une absence prolongée ou ponctuelle d'un leader religieux qu'une opposition s'organise ou lors de son retour que la crise éclate.

27. Ces églises sont par ordre chronologique d'implantation : l'Église apostolique (créée en 1988), Holy Spirit Revival Church (1995), Cornerstone Friends Mission Church (2001), New Hope (2002), New Life Church (2006), Rarotonga Community Church (2008) et Celebration on the Rock (2009). 
s'implanter à Rarotonga. Mais progressivement la frontière s'amenuise à mesure que l'église locale s'internationalise et que l'église d'origine étrangère adopte une stratégie d'intégration locale.

En 1988, alors pasteur depuis 8 ans de la CICC, Tutai Pere décide, suite aux enseignements donnés par un pasteur nord-américain d'une église pentecôtiste en vacances à Rarotonga, de démissionner pour fonder une nouvelle église :

«En 1987, j'ai rencontré un touriste originaire d'Amérique, un homme noir. Deux d'entre eux sont venus et se sont installés à l'hôtel, ils circulaient à vélo autour de l'île et se sont arrêtés à la maison pastorale à Titikaveka, et alors moi et ma femme avons partagé avec eux, sur les Écritures, la Parole et, progressivement, j'ai commencé à apprendre quelques petites choses sur le salut et tout ça. J'ai commencé à évoluer et à être convaincu dans mon cœur (...). Ça a donc été la première influence de ma vie, concernant le pentecôtisme, de la part de ce pasteur des ÉtatsUnis qui est venu ici juste comme touriste. ${ }^{28}$.

L'église du pasteur Pere, aujourd'hui implantée à Rarotonga, Atiu, Mangaia et Aitutaki, devient membre des Pentecostal Assemblies of the World. En septembre 2009, le pasteur Pere est ordonné évêque du diocèse du Pacifique Sud qui inclut les églises apostoliques aux îles Cook, en Nouvelle-Zélande, Australie, Fidji, Vanuatu et Papouasie Nouvelle-Guinée. À la différence de la CICC, l'église apostolique de Rarotonga organise des cultes en langue anglaise pour mieux revendiquer le caractère multiculturel de l'église :

«Dans notre organisation, notre plus grand objectif est d'être international, nous ne nous définissons pas comme une organisation locale. Nous devons mettre en place des congrégations internationales et multiculturelles (...) nous devons nous adapter, alors nous avons ici des Américains, nous avons des Fidjiens, nous avons des Ni-Vanuatu, nous devons parler dans une langue qu'ils comprennent: l'anglais. ${ }^{29}$

Celebration on the Rock est la dernière église implantée à Rarotonga par un couple néo-zélandais, fondateurs d'un réseau d'églises établies en NouvelleZélande, aux États-Unis et en Ouganda, dont Celebration Centre, une megachurch de 2000 personnes à Christchurch en Nouvelle-Zélande désormais dirigée par leurs deux fils ${ }^{30}$. Les fondateurs de Celebration on the Rock, Murray et Nancy Watkinson, développent une approche ciblée sur les valeurs familiales et, comme de nombreuses megachurches nord-américaines [Fath, 2008, p. 71-100], une stratégie de multiplication et de professionnalisation des activités notamment commerciales, sportives et musicales. La stratégie d'implantation locale de Celebration on the Rock consiste d'une part à intégrer des Cook Islanders (précédemment engagés dans différents ministères des Assemblées de Dieu) au sein de l'équipe dirigeante et d'autre part à insister sur l'origine de l'épouse : «Nancy is of

28. Entretien avec Tutai Pere, pasteur de l'Église apostolique à Rarotonga du 7 septembre 2009.

29. Plusieurs centaines de Fidjiens, ni-Vanuatu et Samoans résident temporairement à Rarotonga, où ils constituent une main-d'œuvre bon marché pour les restaurants et les hôtels de l'île.

30. www.celebrationcentre.com 
Rarotongan and Mangaian descent and says she understands this is one of the first churches set up by locals. » (CIN, 15/02/09)

L'héritage chrétien de la nation est inscrit dans le préambule de la Constitution des îles Cook de 1965 qui stipule «The people of the Cook Islands affirm that the Cook Islands is a nation founded on Christian principles, Cook Islands custom, and the rule of law ». Dans ce contexte, le Religious Advisory Council (RAC) a pour mission de conseiller le gouvernement sur les questions religieuses. À l'origine, au début des années 1970, le RAC était composé de quatre églises : la CICC, l'Église catholique, les Adventistes et les Mormons; depuis deux Églises l'ont rejoint : les Assemblées de Dieu et l'Église apostolique. Le RAC, obligatoirement consulté avant que le gouvernement n'autorise une nouvelle Église à organiser des activités aux îles Cook, a refusé la venue de l'Église de l'Unification (secte Moon) en 2005-2006 et en 2008 de l'Église de Scientologie. Les arguments pour refuser l'établissement de ce mouvement religieux sont avant tout d'ordre théologique : il s'agit de prouver que la Scientologie n'est pas chrétienne parce que ses adeptes ne croient pas en Dieu. Pour ce faire, les membres du RAC ont effectué des recherches sur Internet. La lecture de la presse people est également mobilisée : un célèbre acteur américain membre de l'Église de Scientologie est cité comme un exemple inquiétant, illustrant comment l'information - diffusée par les médias néo-zélandais - est réinterprétée pour asseoir une stratégie locale de résistance [Appadurai, 2005, p. 36].

À la différence de Celebration on the Rock, l'église apostolique est membre du Religious Advisory Council :

«Je n'avais pas très envie, au départ »-précise le pasteur Pere - «de faire partie d'un genre d'instance de contrôle bureaucratique, mais si l'on veut être reconnu par toute la population, ça fait partie de la vie sociale, on ne peut pas s'isoler soi-même. Donc c'est la différence qu'il y a entre la foi et les doctrines, c'est un bureau pour la population, quelles que soient les différences, les religions et les croyances. Si le gouvernement a besoin de conseils de la part des églises sur certaines questions, il va voir ce bureau. C'est ce que j'aime là-dedans, parce que je me suis rendu compte que beaucoup de nos prédicateurs locaux sont restés trop silencieux et trop tranquilles sur des questions très controversées (...) donc je voulais être dans ce bureau parce que j'avais pu voir son influence politique... Je veux prendre position sur certaines questions. Alors je suis entré au bureau. »

Cette adhésion au RAC souligne l'acceptation dans la pratique d'un pluralisme religieux dont le principe reste pourtant largement débattu. Cette adhésion est justifiée par des motifs stratégiques - le gouvernement et le parlement sont constitués en « champ de mission »- et culturels : l'identité culturelle et nationale prime sur les appartenances confessionnelles. Néanmoins cette unité culturelle ne vaut que pour rappeler l'identité chrétienne de la nation des îles Cook. C'est cet argument qui est mis en avant pour refuser la venue d'églises non-chrétiennes mais aussi, paradoxalement, pour durcir les conditions d'implantation de nouvelles églises chrétiennes. Le RAC s'est en effet également prononcé pour freiner la venue de Celebration on the Rock, en proposant désormais pour toute nouvelle 
église chrétienne une période probatoire de 5 ans - au cours de laquelle les dirigeants devront établir régulièrement des comptes rendus de leurs activités - avant d'être officiellement reconnue. Le président de la CICC, Tangimetua Tangata Tutai, va plus loin en déclarant: «There are too many small churches in the country, creating confusion amongst our people » (CIN 27/03/09). Cette position du RAC pourrait être interprétée comme une volonté des églises membres de défendre leur «pré-carré ». Elle souligne aussi l'imbrication de différents types de légitimité inégalement répartis entre les différentes églises chrétiennes des îles Cook. Si la CICC peut se permettre, du fait d'une légitimité historique et politique, de se prononcer pour le statu quo, la position de l'Église apostolique apparaît pour le moins ambiguë. Tutai Pere en tant que président du RAC promeut une restriction de la liberté religieuse à Rarotonga alors que dans le même temps, mais sur une autre île, il revendique le droit à la liberté religieuse pour établir une église apostolique à Pukupuka. En effet, dans chaque île existe un RAC «local » réunissant exclusivement les représentants des églises présentes sur l'île. En décembre 2007 lors de la commémoration de « l'arrivée de l'Évangile » sur l'île coïncidant avec l'achèvement de la traduction de la Bible en Pukapuka, les représentants des autorités politiques et religieuses de l'île refusent la venue d'autres confessions :

\begin{abstract}
«The island's member of parliament, Vai Peua, said "Everyone else (church) is banned from the island except for the CICC, SDA [Adventistes] and Catholic. We do not want new religions to come to the island, and we do not want them there for the celebration (...) Head of the Apostolic church in the Cook Islands Pastor Tutai Pere said that the ban placed on his denomination is nothing new at all." (...) Pastor Pere said at one time Rarotonga and Aitutaki placed the same ban but "things changed because one day some of those islanders returned home and wanted to take their faith with them...they will do that in Pukapuka one day". »
\end{abstract}

Mais subrepticement, le dispositif restrictif apparaît défaillant :

«A large delegation of Pukapukans from New Zealand sailed to the island on Sunday and it is understood that some of them belong to religions other than the SDA, CICC and Catholic »

(CIN, 07/12/07)

\title{
Conclusion
}

L'étude des migrations nécessite une approche comparative, dès lors que «le migrant vit une situation de facto comparative » [Green, 2002, p. 5]. Les pratiques religieuses des Cook Islanders en Nouvelle-Zélande suscitent ainsi deux interprétations divergentes selon l'angle comparatif choisi.

La première lecture insiste sur la plus faible pratique religieuse des Cook Islanders de Nouvelle-Zélande par rapport à ceux des îles Cook. La question de l'authenticité des pratiques religieuses prend forme dans l'opposition conversion versus routination. Les responsables des églises pentecôtistes développent ainsi une approche critique de la CICC dont les membres une fois émigrés en Nouvelle-Zélande, en « oubliant » qu'ils sont chrétiens, prouvent le caractère routinier des pratiques religieuses de la CICC : 
"Ils disent haut et fort "nous sommes un pays chrétien" »- note Tutai Pere - " "nous sommes un peuple chrétien, une nation chrétienne", mais dès qu'ils sont loin d'ici, ils l'oublient! (...) Je dirais que c'est une faiblesse de notre Églisemère, la CICC, ils n'équipent pas les fidèles dans la Parole et la doctrine, c'est juste une histoire de routine, juste aller à l'église, rentrer à la maison, ça n'est pas quelque chose de vivant dans leur vie de tous les jours. »

La seconde lecture - à partir d'une comparaison entre les pratiques religieuses des Cook Islanders et celles des Pâkehâ de Nouvelle-Zélande - permet en revanche de souligner le dynamisme relatif des Cook Islanders. Dans un contexte néozélandais où désormais ceux qui se déclarent chrétiens représentent moins de la moitié de la population, l'identité chrétienne devient distinctive et militante [Lineham, 2007]. En retour, ce militantisme génère aux îles Cook un renforcement de la revendication de l'héritage chrétien de la nation et une intensification des projets d'implantation d'églises chrétiennes débouchant sur une gestion restrictive du pluralisme religieux.

Au cours des années 1960 l'imbrication entre identité nationale et identité chrétienne a permis grâce à une définition inclusive du christianisme (tous les Cook Islanders se reconnaissent dans une culture chrétienne) d'ériger les principes chrétiens en valeur constitutionnelle et l'église héritière de la LMS en patrimoine culturel. Désormais la lecture de l'histoire missionnaire et nationale des îles Cook autorise, dans un contexte de forte diversification religieuse, une définition exclusive du christianisme (un «vrai » Cook Islander ne peut fréquenter qu'un certain type d'églises chrétiennes). Cette définition à géométrie variable d'un christianisme culturellement acceptable se traduit par une gestion ambiguë du pluralisme religieux, un terme qui est lui-même l'objet d'interprétations divergentes ; James Beckford [2003] distingue ainsi trois niveaux : la diversité religieuse, la reconnaissance de cette diversité religieuse et le pluralisme comme acceptation positive de cette diversité.

Cette double lecture illustre la facilité avec laquelle les personnes changent d'échelle, utilisent des références à géométrie variable, ce qui complexifie par ailleurs les modes de légitimité religieuse. C'est sans doute pour ces raisons que la catégorie du missionnaire est plébiscitée par de nombreux pasteurs indépendamment du lieu d'exercice ou de l'église d'appartenance.

La figure du missionnaire du fait de son parcours itinérant et de son objectif d'évangélisation incarne en effet l'idéal-type de cette modernité religieuse articulée autour du pèlerin et du converti [Hervieu-Léger, 1999]. Elle montre aussi comment les différences culturelles peuvent être mobilisées au service de l'évangélisation [Fer, 2010, p. 116]. À partir de la typologie wéberienne des différents types d'autorité traditionnelle, institutionnelle et charismatique, Jean-François Zorn définit le champ missionnaire comme une scène sociale où ces trois registres d'autorité sont en conflit et le missionnaire comme le personnage le plus à même d'acquérir une double légitimité, institutionnelle et traditionnelle, "sans se dessaisir de sa liberté d'action» [Zorn, 2004, p. 31]. Enfin la figure contemporaine 
du missionnaire puise aux sources du Réveil évangélique qui en Grande Bretagne a donné naissance à des sociétés missionnaires interdénominationnelles comme la LMS. L'implication de Cook Islanders dans l'évangélisation des îles d'Océanie est partout soulignée avec insistance par les pasteurs des PIC et de la CICC, leur permettant d'inscrire leur parcours migratoire et leur engagement ministériel dans des dynamiques historiques anciennes.

Alors que la gestion du pluralisme religieux relève d'une mise en scène de la diversité chrétienne au service de l'unité nationale, la figure omniprésente du missionnaire illustre comment les différences culturelles peuvent être mises au service de l'évangélisation. La diversité religieuse et culturelle ne devient donc une source de mobilisation que dans la mesure où elle s'inscrit à l'intérieur d'un cadre de référence (la nation) et d'action (l'évangélisation) qui se trouve ainsi renforcé.

\section{Bibliographie}

AlexeyefF K. [2004a], «Sea Breeze : Globalisation and Cook Islands Popular Music », The Asia Pacific Journal of Anthropology, vol. 5, n ${ }^{\circ}$ 2, Août, p. 145-158.

AleXeYefF K. [2004b] «Love Food : Exchange and Sustenance in the Cook Islands Diaspora », The Australian Journal of Anthropology, vol. 15, $\mathrm{n}^{\circ}$ 1, p. 68-79.

ApPadurai A. [2005], Après le colonialisme. Les conséquences culturelles de la globalisation, Payot \& Rivages, Paris.

BARE J.-F. [1987], Tahiti, les temps et les pouvoirs. Pour une anthropologie historique du Tahiti post-européen, Orstom, Paris.

BeCKFord J. [2003], Social Theory and Religion, Cambridge University Press, Cambridge.

BOYER V. [2008], Expansion évangélique et migrations en Amazonie Brésilienne, IRD-Karthala, Paris.

Catarino C., Morokvasic M. [2005], « Femmes, genre, migration et mobilités », Revue Européenne des Migrations Internationales, $\mathrm{n}^{\circ}$ 21/1, p. 7-27.

Connell J., Brown P-C. [2005], Remittances in the Pacific. An Overview, Asian Developement Bank.

Crocombe R., M. [2003], Cook Islands Culture, Akono’Anga Maori, Cooks Islands et Fidji.

FANCELLO S. [2008], « Les pentecôtismes indigènes. La double scène africaine et européenne », Archives de sciences sociales des religions, $\mathrm{n}^{\circ} 143$ (juillet-septembre 2008), p. 69-89.

FAtH S. [2008], Dieu XXL. La révolution des megachurches, Autrement, Paris.

FER Y. [2010], L'offensive évangélique. Voyage au cour des réseaux militants de Jeunesse en Mission, Labor et Fides, Genève.

FRIEDMAN J. [2002], "Y a-t-il un véritable Hawaiien dans la salle ? Anthropologues et "indigènes" face à la question de l'identité », in Hamelin, Wittersheim (dir.), La tradition et l'État, Paris, l'Harmattan.

GreEN N. [2002], Repenser les migrations, PUF, Paris. 
HERVIEU-LÉGER D. [1999], Le pèlerin et le converti. La religion en mouvement, Paris, Flammarion.

HoOPER A. [1961], «Cook Islanders in Auckland», The Journal of the Polynesian Society, vol. 70, n 2, p. 145-193.

Howard A. [1999], « Pacific-Based Virtual Communities : Rotuma on the World Wide Web », The Contemporary Pacific, vol. 11, n ${ }^{\circ}$ 1, p. 160-175.

LANGE R. [2005], Island Ministers. Indigenous Leadership in Nineteenth Century Pacific Islands Christianity, University of Canterbury (New Zealand) et Research School of Pacific and Asian Studies (The Australian National University).

Lineham P. [2007], « Number crunching », Anglican Taonga, Easter, p. 10-12.

MACPHERSON C. et L. [2001] : « Evangelical Religion among Pacific Island Migrants : New Faiths or Brief Diversions ? » Journal of Ritual Studies, vol. 15, University of Pittsburg, États-Unis.

Macpherson C., Spoonley P., Anae M. (eds.) [2001], Tangata O te Moana Nui : The Evolving Identities of Pacific Peoples in Aotearoa/New Zealand, Dunmore Press, Palmerston North, New-Zealand.

MALOGNE-Fer G. [2010], «L'ordination des gays et lesbiennes dans l'église méthodiste de Nouvelle-Zélande : conflits théologiques ou culturels ? », in S. Fath (dir.), Protestantisme évangélique et valeurs, Excelsis, Cléon d'Andran.

MALOGNE-Fer G. [2009a], «Les cultes de langues française et anglaise dans les églises protestantes de Polynésie: intégration des "jeunes" ou pluralisation religieuse? », Social Compass, $\mathrm{n}^{\circ}$ 56/2, p. 249-262.

Malogne-Fer G. [2009b], "Migrations, affiliations religieuses et égalité des sexes en Nouvelle-Zélande », in Fer, Malogne-Fer (dir.), Anthropologie du christianisme en Océanie, L'Harmattan, Paris, p. 103-124.

Morton LeE H. [2004], «All Tongans Are Connected : Tongan Transnationalism », in Lockwood Victoria (ed.), Globalization and Cultural Change in the Pacific Islands, New Jersey, Pearson Prentice Hall, p. 133-149.

Rallu J.-R. [1997], Population, migration, développement dans le Pacifique Sud, UNESCO, Paris.

Sissons, J. [1997] « Nation or Desti-nation ? Cook Islands Nationalism since $1965 »$ in Ton O., et Nicholas T., Narratives of Nation in the South Pacific, Harwood Academic Publishers.

Spoonley P., Macpherson C., Pearson D. (eds.), [2004] Tangata Tangata The changing ethnic contours of New Zealand, New Zealand.

Taule'Ale'Ausumai F. [1997], «The Word Made Flesh : a Samoan Theology of Pastoral Care » in Philip Culbertson (dir.), Counselling Issues \& South Pacific Communities, Auckland, p. 161-187.

Teauariki T. [1996], «My Mission to Papua », in Doug M. et Andrew T., The Covenant Makers. Islander Missionaries in the Pacific, Fidji, p. 255-275.

Thomas N. [1998], Hors du temps. Histoire et évolutionnisme dans le discours anthropologique, Belin, Paris.

ZoRn J.-F. [2004], La missiologie. Émergence d'une discipline théologique, Labor et Fides, Genève. 